\title{
Controlled direct effect of psychiatric disorders on cardiovascular disease: evidence from a large Kurdish cohort
}

Zahra Darabi ${ }^{1}$, Farid Najafi ${ }^{2}$, Roya Safari-Faramani ${ }^{3}$ and Yahya Salimi ${ }^{4 *}$ (]

\begin{abstract}
Background: Psychiatric disorders are significantly associated with the incidence and prevalence of cardiovascular diseases, mortality, hospital readmissionn. Oral and dental hygiene may play a role in such association. This study aimed to evaluate the controlled direct effect of psychiatric disorders on cardiovascular diseases by controlling the mediating effect of oral and dental hygiene.

Methods: The data used for this study came from the baseline phase of Ravansar Non-communicable Disease (RaNCD) cohort study. RaNCD cohort study is including a representative sample of 10,065 adults (35-65 years old) living in Ravansar, a city in the west of Iran. The marginal structural model with stabilized inverse probability weights accounted for potential confounders was used to estimate the controlled direct effect of psychiatric disorders on cardiovascular diseases. Three different models using three mediators including oral and dental hygiene behaviors, oral ulcer and lesions, and decayed, missing, and filled tooth, were used.
\end{abstract}

Results: Psychiatric disorders increase the odds of cardiovascular diseases by $83 \%(\mathrm{OR}=1.83, \mathrm{Cl} 1.27,2.61)$ and about two times $(\mathrm{OR}=2.14,95 \% \mathrm{Cl} 1.74,2.63)$ when controlled for oral and dental hygiene behaviors, and oral ulcer and lesions as mediators, respectively. When decayed, missing, and filled tooth, as a mediator, was set at $\leq 8$, there was no statistically significant controlled direct effect of psychiatric disorders on cardiovascular diseases $(\mathrm{OR}=0.90,95 \% \mathrm{Cl}$ $0.62,1.30)$.

Conclusion: Our results suggested that psychiatric disorder was directly related to cardiovascular diseases even if it was possible to have good oral and dental hygiene. The results suggested that interventions targeting people with psychiatric disorders could reduce prevalence of the cardiovascular diseases.

Keywords: Cardiovascular disease, Psychiatric disorders, Oral and dental hygiene, Controlled direct effect

\section{Background}

Cardiovascular disease (CVD) is the leading cause of death and morbidity worldwide and accounting globally for around $30 \%$ of all deaths [1]. Many effective interventions for primary and secondary prevention of heart

\footnotetext{
*Correspondence: salimiyahya@yahoo.com

${ }^{4}$ Social Development and Health Promotion Research Center, Health Institute, Kermanshah University of Medical Sciences, 6719851351 Kermanshah, Iran

Full list of author information is available at the end of the article
}

disease rely on identifying people at risk. Despite the reliability of risk prediction models, a significant proportion of CVD cases occur in individuals without known risk factors [2]. Studies have shown that negative emotions, along with psychiatric disorders such as depression, can increase the CVD incidence and mortality [3-5]. Indeed, in people with depression, it is twice as likely to develop myocardial infarction as compared with the general population [6, 7]. Death from heart disease among depress people occurs even more than suicide deaths [6]. 
Several factors may have a role in the relationship between depression and CVD. Oral and dental hygiene is an indicator of general health and quality of life [8]. Such factors can affect quality of life through functional impairment, pain, discomfort and disability. Oral and dental problems are constantly highlighted in the list of common disease, but they are still referred to as silent epidemics and are therefore frequently ignored in public health planning $[9,10]$. It has been shown that poor oral and dental hygiene including periodontal disease and teeth decay are associated with an increased risk of CVD [11, 12]. A review of 42 self-reported studies in 2012, revealed a correlation between periodontal disease and, atherosclerotic vascular disease [11]. Also, the harmful effects of mental disorders on organic control of human tissues, known as psychosomatic disorders, affect both periodontium and oral cavity in two ways: first, by creating unhealthy habits that are destructive for periodontal invasion, and secondly through the direct effect of the nervous system on the physiological balance of tissue [13]. Although there is large number of studies on the risk factors of CVD, but research on interaction between factors such as depression, oral and dental hygiene, and their mechanism are scarce $[11,14]$. Therefore, we aimed to disentangle the direct effect of psychiatric disorder on CVD, controlling for oral and dental hygiene using a large sample of Ravansar Non-Communicable Disease (RaNCD) cohort study.

\section{Methods}

The data consists of 10,065 individuals aged 35-65 years old from first cohort study in Kurdish region. In fact, RaNCD cohort study is one of the centers of the nationwide Prospective Epidemiological Research Studies in IrAN (PERSIAN). Inclusion criteria for participant recruitment were: age 35-65 years, minimum residence of 1 year in Ravansar city, likelihood of staying in Ravansar city in future, the willingness to participate and provision of written informed consent; having Iranian citizenship. After a census from total population of Ravansar district, 10,000 people aged 35-65 years old were sampled from both urban and rural areas. Data were collected using trained research assistants, who had good communication in Kurdish and local languages, conducted a door-to-door survey of all residents in urban areas to register their home address and to specify a code for each household. In the rural areas, local health units (Health Houses) already had all registration details for all residents. Household members' details, including name, age and relationship, were registered, along with their contact number. All the process, questionnaires and forms for data collection have been verified by a central team responsible for designing and conduction the PERSIAN cohort study. Details of the sampling techniques, rational and methods are described elsewhere $[15,16]$.

\section{Measures}

The information required for conducting this study was collected by standard questionnaires and by trained experts in the RaNCD cohort study. After the pre-pilot, measuring tools and protocols were revised to improve the validity and reliability of the study, as well as to streamline data collection. While the central team of PERSIAN cohort supervise all centers by a quality control team, the quality of all measurements and process are under supervision of an epidemiologist responsible for quality control in RaNCD. Although most of measurements have been collected by self-report, the medical history and the type of received treatments have been checked by interviewers and general physicians. Validity and reliability of some of measurements have been reported elsewhere [17-20]. The principal investigators decided to use the online version of the questionnaire, in accordance with the PERSIAN cohort protocol [16]. Procedures for data access, information on collaborations, questionnaire information, publications and other details can be found at http://persiancohort.com

\section{Exposure}

Data on psychiatric disorders was collected based on self-report of any physician diagnosis. In addition, after consultation with psychiatrists, any medications related to psychiatric disorders were separated as psychiatric disease. Finally, the variable of psychiatric disorder was made up of a combination of four variables, including self-reported depression (diagnosed by a physician), taking antidepressant medication, self-reported other psychiatric disorders (diagnosed by a medical doctor), and taking medications for other psychiatric disorders.

\section{Outcome}

Participants who had at least 1 of the following condition were classified as patients with CVD: a history of ischemic heart disease, heart failure and angina, stroke, and/or use of relevant medications for such condition. A list of used medications was also approved by consulting with an internal medicine specialist. In other words, people with CVD were those who either had a diagnosis of heart disease according to their statements by the doctor or had approved medicine for heart disease in their list of medicine.

\section{Mediators}

Oral and dental hygiene was measured by asking the number of decayed, filled and missing teeth, oral lesions 
and ulcers, and hygiene behaviors; including frequency of daily teeth brushing, using dental floss and mouthwash. We used this data to create 3 oral and dental hygiene indices. Good oral hygiene behaviors were defined as cleaning teeth by brushing at least twice and flossing once daily [21]. We used self-reported data to evaluate the variable of ulcer and oral lesions. Decayed, missing, filled tooth (DMFT) was categorized to mild decay $(\mathrm{DMFT} \leq 8)$ and severe decays $(\mathrm{DMFT}>8)[22]$.

\section{Covariates}

Demographic data including age and sex and socioeconomic status (SES) was collected by trained interviewers. The SES was measured by an asset index, constructed using principal component analysis from data on durable household assets [23, 24].

The blood pressure was measured using a manual sphygmomanometer (Riester). From each arm, blood pressure measured two times (with an interval of $10 \mathrm{~min}$ ) and the average of two measurements was the final blood pressure of the participants. Data on smoking, alcohol and narcotics were collected, using questionnaires for personal habits (use of alcohol and tobacco). People who smoked more than 100 cigarettes in their lifetime or who were smoking daily, considered as smokers.

To measure and evaluate the physical activity, we used the International Physical Activity Questionnaire (IPAQ), which included questions about physical activity in the last 7 days [25]. The total Metabolic Equivalent of Task (METs) was calculated according to the questionnaire's instructions. For this study, physical activity was categorized to three-level: low physical activity (less than the 36.5 Met-hour/week), medium physical activity (between 36.6 and 44.9 Met-hour / week) and group with high physical activity (45 Met-hour/week and more) [26]. Levels of plasma total cholesterol and triglyceride were measured enzymatically.

The evaluation of food intake for each individual was done, using the Food Frequency Questionnaire (FFQ), which its validity and reliability previously was approved [25]. The questionnaire consisted of a list of 168 food items with standard portion size. The amounts indicated for each meal were converted to the recommended daily intake [27]. In this study, the variables of sugar and dairy intake were used in the form of g/day and vitamin $\mathrm{D}$ intake as $\mu \mathrm{g} /$ day. To evaluate the variables of sleep, the 24-h sleep time was calculated.

\section{Data cleaning}

First, the data were modified in terms of the names of variables, codes and labels related to each variable and their unit of measurement. Then the qualitative data were identified and converted into appropriate codes based on the objectives of the plan. Then, for each variable, the outgoing data were identified and modified. First, the natural range of the data was determined. Then the data whose value was higher than expected became the highest natural number and the data whose value was lower than expected became the lowest natural number for the variable.

\section{Exposure-induced mediator-outcome confounders}

We used the Directed Acyclic Graph (DAG) for variable selection (Fig. 1). let L denote oral and dental hygieneCVD confounders affected by the psychiatric disorder (including age, sex, high blood pressure, diabetes mellitus, physical activity, smoking, drugs and alcohol, blood cholesterol, SES, body mass index (BMI), level of education and diet), $C_{1}$ is exposure-mediator confounders and $\mathrm{C}_{2}$ is exposure-outcome confounders. In the presence of exposure-induced mediator-outcome confounders, natural direct and indirect effects are not identified [28].

In this study, we estimated the controlled direct effect $(\mathrm{CDE})$ using the marginal structural models under the following assumptions. First, the $\mathrm{C}_{2}$ variables are sufficient to eliminate the confounding effect in the relationship between psychiatric disorder and CVD. Second, $C_{1}$ and $L$ variables are sufficient to control the confounding effect in the relationship between the oral and dental hygiene and CVD [29]. This model does not directly use regression methods, but instead uses a weighting method called "inverse probability weighting".

The base of marginal structural models is to analyze the regression model $\mathrm{Y}$, as outcome, on $\mathrm{E}$, as exposure, and $\mathrm{M}$, as mediator, but instead of controlling the confounders $\mathrm{C}$ and $\mathrm{L}$ with regression, they are controlled by weighting approach [30,31]. Therefore, two sets of weights are needed: one for the psychiatric disorder as exposure $\left(W_{i}^{E}\right)$ and one for the oral and dental hygiene as mediator $\left(W_{i}^{M}\right)$. In addition, two important assumptions for the estimation of CDE are: (1) the relationship between psychiatric disorder and CVD is dependent on $\mathrm{C}_{2}$ (2) the relationship between the oral and dental hygiene and CVD depends on $\mathrm{C}_{1}$ and L. Marginal Structural Models for a single mediator model allowing interaction between exposure and mediator is written as follows:

$$
E[Y \mid a, m]=\gamma_{0}+\gamma_{1} e+\gamma_{2} m+\gamma_{3} e m
$$

In this study, each participant (i) is weighted by the total weight of exposure and mediator: $W_{i}=W_{i}^{E} \times W_{i}^{M}$. Then, the controlled direct effect can be obtained based on the estimates using such weighting approach. To calculate the $\mathrm{CDE}$ of $\mathrm{E}$ on $\mathrm{Y}$ when the mediator $\mathrm{M}=\mathrm{m}$, the following formula will be used if the exposure change from the level $\mathrm{e}^{*}$ to the level e: 


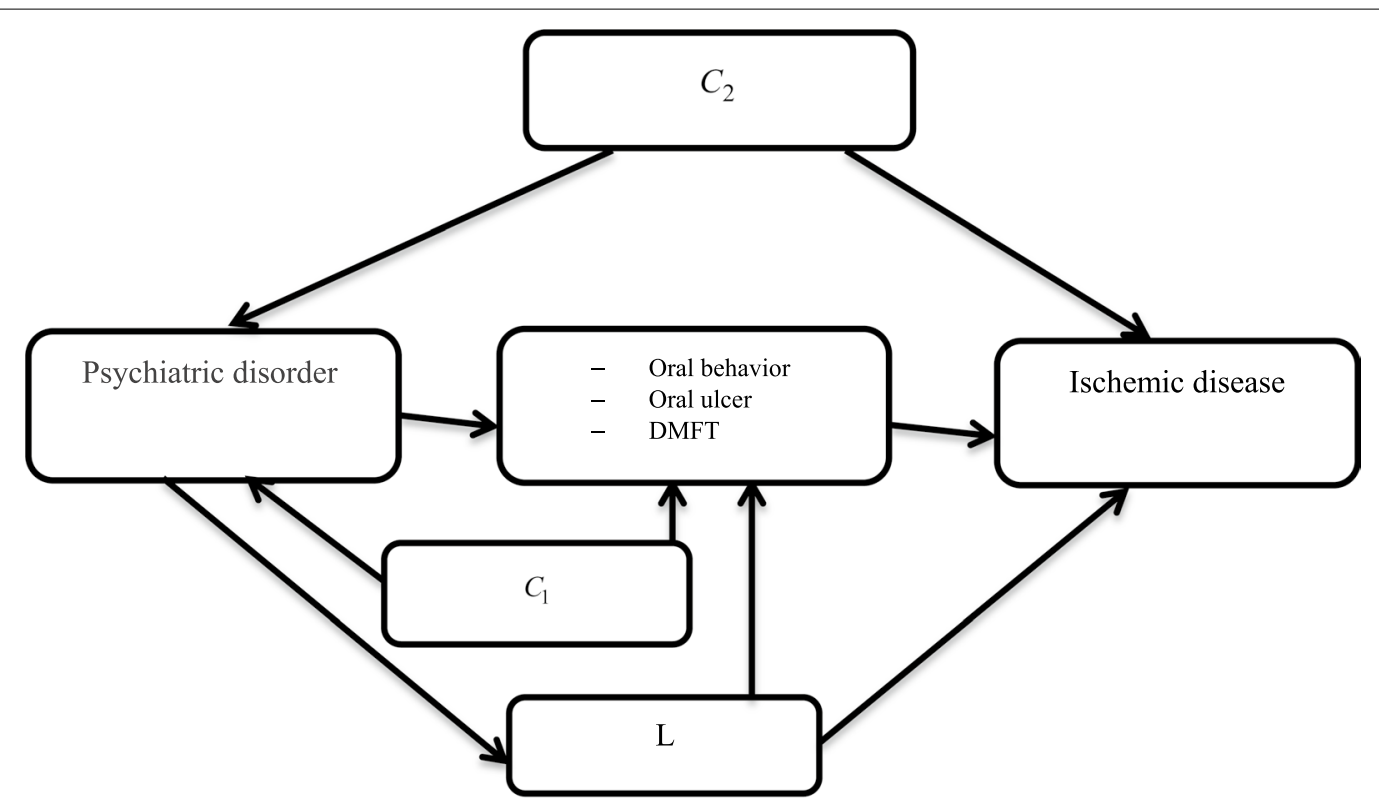

Fig. 1 DAG of the hypothesized causal pathway between psychiatric disorder and CVD. DMFT: Decayed, Missing, Filled Tooth, DAG: Directed Acyclic Graph, $c_{1}$ (confounder1): Age, Sex, Smoking, Educational, Diabetes mellitus, C (confounder2): Age, Sex, Smoking, BMl, Physical activity, Diet, Sleep time, L (Exposure-induced mediator-outcome confounders): Age, sex, Hypertension, Diabetes mellitus, Physical activity, Drinking, Hypercholesterolemia, SES, education, diet, BMI, Smoking, Drug use, Sugar use

$$
\begin{aligned}
& C D E(m)=\gamma_{1}\left(e-e^{*}\right)+\gamma_{3} m\left(e-e^{*}\right) \\
& \text { and } e^{*}=0 \text { and } e=1
\end{aligned}
$$

so, the formula can be written as follows:

$$
C D E(m)=\gamma_{1}+\gamma_{3} m
$$

\section{Sensitivity analysis}

Inappropriate classification may overestimate the association between psychiatric disorder and CVD. Sensitivity analysis was used to measure the strength of the findings. Therefore, we conducted two sensitivity analyzes: one for different definitions of exposure, and another for various mediator definitions that were found to be consistent with our findings.

We also initially performed all analyzes, using primary data that included missing data. The data were then analyzed after multiple imputation. The maximum missing data were $2.79 \%$. The results of both analyzes were similar. We decided to present the results of primary data including missing.

\section{Data analysis}

Descriptive statistics were used for describing the data. The marginal structural model was used to estimate controlled direct effect (CDE) in the presence of interaction between psychiatric disorder and, oral and dental hygiene [29]. The controlled direct effect is the effect of psychiatric disorder through pathways that do not pass through oral and dental hygiene status, holding oral and dental hygiene status at a constant level across all individuals. In this study, our outcome variable was a binary variable. The mediator variables were both binary and quantitative. We introduced the categorical factors as dummy variables. Records with missing data were excluded because the amount of missing data was small less than $1 \%$ and assumed to be missing at random. All analyses were performed using codes provided by VanderWeele et al. [32] in Stata11 (StataCorp, College Station, TX, USA).

\section{Results}

From total of 10,065 participants of RaNCD cohort study, $5200(52.5 \%)$ were female, 2496 (24.7\%) were illiterate and 9076 (90.2\%) were married (Table 1). Cardiovascular disease (angina, myocardial infarction, stroke, transient ischemic attack) was detected in $13.73 \%$ (1382 people) of the study population. The proportion of CVD among the females $(17.70 \%)$ was higher compared to the males (10.37\%). People who were over 45 years had a higher prevalence of CVD. Also, people with lower levels of education and lower socioeconomic conditions had a higher prevalence of CVD.

Among people with CVD, 90.17\% were married, 9.18\% divorced, and $0.64 \%$ were bachelor. Among people with CVD $8.91 \%$ were smokers and $12 \%$ were former smokers. 
Table 1 Description of cardiovascular diseases in subgroups of the independent variables

\begin{tabular}{|c|c|c|c|c|}
\hline Independent variables & $\begin{array}{l}\text { With CVD } \\
\text { n (\%) }\end{array}$ & $\begin{array}{l}\text { Without CVD } \\
\text { n (\%) }\end{array}$ & $\begin{array}{l}\text { Total } \\
\text { n (\%) }\end{array}$ & Odds ratio $(95 \% \mathrm{Cl})$ \\
\hline \multicolumn{5}{|l|}{ Gender } \\
\hline Female & $920(64.47)$ & $4280(49.42)$ & $5200(52.54)$ & $1.80(1.52,2.13)$ \\
\hline Male & $507(35.53)$ & $4380(50.58)$ & $4887(47.46)$ & \\
\hline \multicolumn{5}{|l|}{ Age } \\
\hline$<45$ years & $223(15.87)$ & $3777(43.61)$ & $4000(39.58)$ & $1.05(1.04,1.06)$ \\
\hline$\geq 45$ years & $1182(84.13)$ & $4883(56.39)$ & $6065(60.42)$ & \\
\hline \multicolumn{5}{|l|}{ Years of education } \\
\hline Illiterate & $570(39.94)$ & $1926(22.24)$ & $2496(24.74)$ & $1.02(0.93,1.11)$ \\
\hline $1-5$ years & $525(36.79)$ & $3325(38.39)$ & $3850(38.17)$ & \\
\hline $6-9$ years & $170(11.91)$ & $1511(17.44)$ & $1681(16.67)$ & \\
\hline $10-12$ years & $101(7.07)$ & $1171(13.52)$ & $1272(12.61)$ & \\
\hline$>13$ years & $61(4.27)$ & $727(8.39)$ & $788(7.81)$ & \\
\hline \multicolumn{5}{|l|}{ Marital status } \\
\hline Married & $1267(90.17)$ & $7809(90.17)$ & $9076(90.18)$ & $1.17(1.06,1.30)$ \\
\hline Divorced & $129(9.18)$ & $438(5.05)$ & $567(5.63)$ & \\
\hline Bachelor & $9(0.64)$ & $413(4.76)$ & $422(4.19)$ & \\
\hline \multicolumn{5}{|l|}{ Socioeconomic status } \\
\hline 1 & $326(22.95)$ & $1681(19.52)$ & $2007(20.1)$ & $1.06(1.00,1.13)$ \\
\hline 2 & $298(20.98)$ & $1707(19.82)$ & 2005 (19.99) & \\
\hline 3 & $286(20.14)$ & $1720(19.97)$ & $2006(20.0)$ & \\
\hline 4 & $273(19.22)$ & $1733(20.12)$ & $2006(20.0)$ & \\
\hline 5 & 237 (16.69) & $1769(20.54)$ & $2006(20.0)$ & \\
\hline \multicolumn{5}{|l|}{ Smoking status } \\
\hline No & $1100(79.07)$ & $6927(80.13)$ & 8027 (79.99) & $1.10(0.86,1.40)$ \\
\hline Current & $124(8.91)$ & 1055(12.20) & $1179(11.75)$ & \\
\hline Former & $167(12.00)$ & $662(7.65)$ & $829(8.26)$ & \\
\hline \multicolumn{5}{|l|}{ Drinking } \\
\hline Yes & $95(6.75)$ & $540(6.23)$ & $635(6.30)$ & $1.24(0.91,1.68)$ \\
\hline No & $1311(93.25)$ & $8119(93.77)$ & $9430(93.7)$ & \\
\hline \multicolumn{5}{|l|}{ Druguse } \\
\hline Yes & $39(2.77)$ & $260(3.00)$ & $299(2.97)$ & $1.01(0.63,1.60)$ \\
\hline No & $1367(97.23)$ & $8399(97.00)$ & $9766(97.03)$ & \\
\hline \multicolumn{5}{|l|}{ Physical activity } \\
\hline High & $292(20.76)$ & $1825(21.08)$ & $2117(21.03)$ & $1.00(0.99,1.00)$ \\
\hline Moderate & $697(49.57)$ & $4250(49.09)$ & $4947(49.17)$ & \\
\hline Low & $417(29.65)$ & $2582(29.82)$ & $2999(29.80)$ & \\
\hline \multicolumn{5}{|l|}{ Body mass index (BMI) } \\
\hline$<18 / 5$ & $25(1.79)$ & $152(1.76)$ & $177(1.78)$ & $1.01(1.00,1.03)$ \\
\hline $18 / 5-24 / 9$ & $362(25.96)$ & $2380(27.71)$ & $2742(27.48)$ & \\
\hline $25-29 / 9$ & $606(43.47)$ & 3739(43.53) & $4345(43.52)$ & \\
\hline$\geq 30$ & $401(28.76)$ & $2317(26.97)$ & $2718(27.22)$ & \\
\hline \multicolumn{5}{|l|}{ Hypertension } \\
\hline Yes & $878(62.80)$ & $697(8.06)$ & 1575 (15.69) & $12.78(11.06,14.77)$ \\
\hline No & $520(37.19)$ & 7945 (91.93) & $8465(84.31)$ & \\
\hline \multicolumn{5}{|l|}{ Diabetes mellitus } \\
\hline Yes & $273(19.51)$ & $546(6.34)$ & 819 (8.19) & $2.19(1.79,2.67)$ \\
\hline No & $1126(80.48)$ & $8054(93.65)$ & $9180(91.81)$ & \\
\hline
\end{tabular}


Table 1 (continued)

\begin{tabular}{|c|c|c|c|c|}
\hline Independent variables & $\begin{array}{l}\text { With CVD } \\
\text { n (\%) }\end{array}$ & $\begin{array}{l}\text { Without CVD } \\
\text { n (\%) }\end{array}$ & $\begin{array}{l}\text { Total } \\
\text { n (\%) }\end{array}$ & Odds ratio $(95 \% \mathrm{Cl})$ \\
\hline \multicolumn{5}{|l|}{ Serum cholesterol } \\
\hline$\geq 200$ & $956(66.06)$ & $5864(67.71)$ & $6820(32.52)$ & $1.00(0.99,1.00)$ \\
\hline$<200$ & $491(33.93)$ & $2796(32.28)$ & $3287(67.48)$ & \\
\hline \multicolumn{5}{|l|}{ Serum triglyceride } \\
\hline$\geq 150$ & $968(69.24)$ & $5873(68.27)$ & $6841(68.41)$ & $0.99(0.99,0.99)$ \\
\hline$<150$ & $430(30.75)$ & $2729(31.72)$ & $3159(31.59)$ & \\
\hline \multicolumn{5}{|l|}{ Renal failure } \\
\hline Yes & $17(1.21)$ & $84(0.97)$ & $101(1)$ & $0.90(0.47,1.73)$ \\
\hline No & $1387(98.78)$ & $8566(99.02)$ & 9953 (99) & \\
\hline
\end{tabular}

Table 2 Controlled direct effects of psychiatric disorders on CVD, using different mediators

\begin{tabular}{llll}
\hline Mediators & Odds ratio & \multicolumn{2}{l}{$\mathbf{9 5 \%} \mathbf{C l}$} \\
\cline { 3 - 4 } & & LL & UL \\
\hline Oral behavior & 1.83 & 1.27 & 2.61 \\
Oral ulcer & 2.14 & 1.74 & 2.63 \\
$\begin{array}{l}\text { Decayed, missing, filled } \\
\text { tooth (DMFT) }\end{array}$ & 0.90 & 0.62 & 1.30 \\
& & &
\end{tabular}

About $6.75 \%$ of patients were alcoholics. Also, 2.77\% of patient were drug user. Among people with CVD, 20.76\% had high physical activity, $28.76 \%$ were obese, $62.80 \%$ had hypertension, $19.51 \%$ had diabetes mellitus, $66.06 \%$ suffer from high serum cholesterol, $69.24 \%$ with high serum triglyceride and $1.21 \%$ had renal failure. The prevalence of CVD was also higher among those with obesity (28.76\%) (Table 1).

Our mediation analysis showed that the CDE of psychiatric disorder on CVD controlling the oral hygiene behaviors at the population level (all have a good hygiene behavior), increases the odds of CVD by $83 \%(\mathrm{OR}=1.83$, $95 \%$ CI: $1.27,2.61)$. In the sensitivity analyses, our findings did change. Using oral ulcer and lesions as a mediator, the CDE of psychiatric disorder on CVD was 2.14 (95\% CI: 1.74, 2.63). However, controlling for DMFT as a mediator did not show a significant effect $(\mathrm{OR}=0.90$, 95\% CI: 0.62, 1.30) (Table 2).

\section{Discussion}

In this study, the role of oral and dental hygiene as a mediator of the association between psychiatric disorders and cardiovascular disease, in the population of RaNCD cohort study was investigated. Psychiatric disorders had a direct effect on CVD, controlled for well-known mediators [33]. Although the precise nature of the links between depression and coronary heart disease (CHD) have not yet been established, these links are being highlighted more and more frequently, first of all from an epidemiological point of view and then with regard to the etiological and clinical aspects in patients with both condition [34]. In fact, the thoughtful statistical analysis and the study design are among the major strengths of the present study. The analysis was based on the most recent advances in causal inference methods, and sensitivity analyses were conducted to test for inappropriate classification and missing data. In this study, the prevalence of CVD was $13.73 \%$, which is significantly different from the national average of $32.2 \%$ [34]. This contrast can be due to differences in the definition of disease in studies. Our study evaluated CVD based on self-reporting and used medication, while the definition of disease in the study by Forouzanfar et al. [34] was based on the checklist. Also, this difference could be partly due to lower risk factors for heart disease and healthy lifestyle among the population of RaNCD cohort study.

According to the World Health Organization (WHO), lifestyle is a combination of behavioral patterns and individual habits throughout life, including nutrition, physical activity, stress, smoking, and the quality of sleep, which are due to socialization. In Ravansar, due to its traditional texture and cultural issues, many of these behavioral patterns have not changed in the same way as in large industrial cities. In this study, the prevalence of psychiatric disorders was $7.78 \%$. Based on the results of the second national study on mental disorders in Iran, the estimated prevalence of mood disorders and anxiety disorders were $4.35 \%$, and $8.31 \%$, respectively [35], which is in agreement with ours. Studies have also been conducted in the regional and provincial levels. In a study conducted in Kermanshah province by Sadeghi et al. [36], which was conducted by screening and psychiatric interviewing questionnaire, the periodic prevalence of 
depressive disorder was $1.6 \%$, which is in contrast to the results of this study. Part of this difference is due to the time of the study. Our study was conducted many years after the study be Sadeghi et al. [36], and the prevalence of the depressive disorder has changed considerably over such period. There are other reasons contributing to such differences in the results which variation in age of participants plus use of different questionnaire are two important factors

In this study, a controlled direct effect between psychiatric disorders and CVD was determined. This effect was estimated to be 1.83 while controlling for oral and dental hygiene behaviors. That is if the variable of oral and dental hygiene remains constant and the psychiatric disorders reaches from the level $\mathrm{a} *=0$ to $\mathrm{a}=1$ level, then the risk of cardiovascular disease will be 1.83 -fold. After the control of oral ulcer and lesions, the direct effect of psychiatric disorders was estimated to be 2.14, which was statistically significant. That means if the variable of oral ulcer and lesions stays constant and the variable of psychiatric disorders change from a level of $a *=0$ to $a=1$ level, then the risk of developing the cardiovascular disease will be 2.14 times higher. This is due to the presence of the problem and impairment of the internal tissue of the mouth, which has a direct and strong connection with the blood and vascular system and ultimately can directly affect the cardiovascular function. Also, oral lesions are directly related to the mental status of individuals and the presence of impaired psychologic function can lead to problems in the oral cavity. Some systemic conditions, such as diabetes and physical-psychiatric disorders, such as depression and psychological stress, affect the periodontium. Psychosocial stresses have an important role in periodontal disease if they affect the host's immune response.

In addition, the controlled direct effect of psychiatric disorders on CVD, controlling the DMFT variable, is estimated to be 0.90 . This means that if the DMFT variable remains constant and the psychiatric disorders change from $\mathrm{a} *=0$ to the level $\mathrm{a}=1$, then the risk of cardiovascular disease is reduced to $10 \%$, which is not statistically significant. Logistic regression analysis showed that the low frequency of brushing is significantly associated with a high prevalence of $\mathrm{DM}(\mathrm{OR}=2.03)$. This indicates that the low frequency of brushing leads to an increase in the decayed and missing teeth, which in turn leads to an increase in the incidence of CVD. As a result, the reason for the mild estimation obtained in this section is that people who have been suffering from oral and dental problems and consequently, CVD, due to their noncompliance with oral and dental hygiene, by their own decision or the physician's recommendation, they have changed their behavior and reduced their cardiovascular problems. The findings of this study indicate that psychiatric disorders have a direct impact on CVD in midlife. This study helps to better understand the determinants of cardiovascular disease. Previous studies have shown that poorer oral health, especially dental problems, is an independent predictor of morbidity and mortality from cardiovascular disease [12, 37]. Even after extensive adjustment for recognized confounders, edentate participants had a significantly elevated risk of all-cause and CVD mortality, but not cancer mortality. This highlights the importance of oral health as a mediator in the direct link between psychiatric disorders and cardiovascular disease. When we keep oral health constant, it means that despite considering this variable as a mediator, we can still prove the strong effect of oral health on cardiovascular disease. This strong association between mental illness and cardiovascular disease has been proven in previous studies [38]. However, the direct controlled effect of psychiatric disorders on cardiovascular disease through oral health has not been previously reported in any article. Our findings confirm the previous evidence of the effects of mental health problems on chronic illnesses and suggest that reducing mental health problems may have lifelong benefits for the health of other organs of the body.

\section{Strengths and limitations}

For the purpose of this study, we used baseline data from large cohort of Kurdish people. We also used causal inference methods for mediator analysis that could resolve the limitations of traditional mediation methods. Although the bias due to unmeasured confounding cannot be ruled out, we used DAG to find the all known variables that may be a confounder in the relationships. We assume that our marginal structural models were correctly specified and that the assumption holds. There are several limitations of our study. First, examinations for calculating oral and dental health have some drawbacks: the number of teeth in danger is not seen in this index, the reason for the loss of teeth is not easily detectable; the filled part is affected by the decision of the clinician to fill the tooth. Nowadays, in most cases, restorations are carried out with tooth-colored materials that are difficult to distinguish from the natural tissue of the tooth in epidemiological studies. Second, because of the cross-sectional nature of the data, it was not possible to establish temporality between exposure, mediator and outcome. The current evidence suggest that the association of CVD and psychiatric disorder is bidirectional, psychiatric disorder can cause CVD and CVD can cause psychiatric disorder $[7,39]$. Third, we assume no unmeasured confounding of the exposure-outcome and mediator-outcome association. However, both CVD and psychiatric disorder may 
have a common cause such as inflammatory biomarkers that not studied in the current study. Fourth, the selfreported nature of psychiatric disorders and cardiovascular disease might lead to recall bias. Although, we have verified diagnosis for self-reporting data, using the list of taken medications.

\section{Conclusion}

We demonstrate that psychiatric disorders are directly related to cardiovascular disease. In the optimal situation of hygiene behaviors and oral lesions in the presence of psychiatric disorders, there was a higher probability of having CVD, compared with the absence of psychiatric disorders. These results suggested that interventions targeting people with psychiatric disorders could reduce prevalence of the CVD.

\begin{abstract}
Abbreviations
CVD: Cardiovascular disease; RaNCD: Ravansar Non-communicable Disease; PERSIAN: Prospective Epidemiological Research Studies in IrAN; DMFT:

Decayed, missing, filled tooth; SES: Socioeconomic status; IPAQ: International physical activity questionnaire; MET: Metabolic equivalent of task; FFQ: Food frequency questionnaire; DAG: Directed acyclic graph; BMI: Body Mass Index; CDE: Controlled direct effect; CHD: Coronary heart disease; WHO: World Health Organization; DM: Diabetes mellitus; OR: Odds ratio.
\end{abstract}

\section{Acknowledgments}

The authors thank staff Ravansar Non-communicable disease cohort at Kermanshah Medical University.

\section{Authors' contributions}

YS conceived and supervised the study and performed statistical analysis of the data. ZD was involved in the procedure and wrote the main body of the manuscript. FN participated in the discussion on the interpretation of the research content and took part in the procedure. RS revised this paper. All authors critically revised and approved the final version of the manuscript. All authors read and approved the final manuscript.

\section{Funding}

The study was supported by the Kermanshah University of Medical Sciences. This research Project Number is 97329.

\section{Availability of data and materials}

The datasets used during the current study are available from the corresponding author on reasonable request.

\section{Ethics approval and consent to participate}

All participants provided written consent before entering the study. The study was approved by the Ethics Committee of Kermanshah University of Medical Sciences, with the ethical Approval Number: IR.KUMS.REC.1397.252.

\section{Consent for publication}

Not applicable.

\section{Competing interests}

All authors declare that they have no competing interests.

\section{Author details}

${ }^{1}$ Department of Epidemiology, School of Public Health, Kermanshah University of Medical Sciences, 6719851351 Kermanshah, Iran. ${ }^{2}$ Research Center for Environmental Determinants of Health, Health Institute, Kermanshah University of Medical Sciences, 6719851351 Kermanshah, Iran. ${ }^{3}$ Department of Epidemiology, Research Center for Environmental Determinants of Health, Health Institute, Kermanshah University of Medical Sciences,
6719851351 Kermanshah, Iran. ${ }^{4}$ Social Development and Health Promotion Research Center, Health Institute, Kermanshah University of Medical Sciences, 6719851351 Kermanshah, Iran.

Received: 13 June 2020 Accepted: 24 November 2020

Published online: 01 December 2020

\section{References}

1. Murray CJ, et al. Disability-adjusted life years (DALYs) for 291 diseases and injuries in 21 regions, 1990-2010: a systematic analysis for the Global Burden of Disease Study 2010. Lancet. 2012;380(9859):2197-223.

2. Helfand $M$, et al. Emerging risk factors for coronary heart disease: a summary of systematic reviews conducted for the US Preventive Services Task Force. Ann Intern Med. 2009;151 (7):496-507.

3. Hemingway H, Marmot M. Evidence based cardiology: psychosocial factors in the aetiology and prognosis of coronary heart disease: systematic review of prospective cohort studies. BMJ Br Med J. 1999:318(7196):1460.

4. Lett HS, et al. Depression as a risk factor for coronary artery disease: evidence, mechanisms, and treatment. Psychosom Med. 2004;66(3):305-15.

5. Rozanski A, Blumenthal JA, Kaplan J. Impact of psychological factors on the pathogenesis of cardiovascular disease and implications for therapy. Circulation. 1999;99(16):2192-217.

6. Chaddha A, et al. Mental health and cardiovascular. Disease. 2016:129(11):1145-8.

7. De Hert M, Detraux J, Vancampfort D. The intriguing relationship between coronary heart disease and mental disorders. Dial Clin Neurosci. 2018;20(1):31-40

8. Petersen PE, Kwan S. The 7th WHO Global Conference on Health Promotion-towards integration of oral health (Nairobi, Kenya 2009). Community Dent Health. 2010;27(Suppl 1):129-36.

9. Spencer AJ. What options do we have for organising, providing and funding better public dental care? Sydney: Australian Health Policy Institute Sydney; 2001

10. Health UDO, Services H. Oral health in America: a report of the Surgeon General. London: NIH Publication; 2000. p. 155-88.

11. Lockhart $\mathrm{PB}$, et al. Periodontal disease and atherosclerotic vascular disease: does the evidence support an independent association? A scientific statement from the American Heart Association. Circulation. 2012;125(20):2520-44.

12. Watt $R G$, et al. Tooth loss and cardiovascular disease mortality risk-results from the Scottish Health Survey. PLoS ONE. 2012;7(2):e30797.

13. Grossi S, et al. Microbiological risk indicators for periodontal disease. J Dent Res. 1993;72:206-10.

14. Mucci $L A$, et al. Do genetic factors explain the association between poor oral health and cardiovascular disease? A prospective study among Swedish twins. Am J Epidemiol. 2009;170(5):615-21.

15. Eghtesad S, et al. The PERSIAN cohort: providing the evidence needed for healthcare reform. Arch Iran Med. 2017;20(11):691.

16. Poustchi $\mathrm{H}$, et al. Prospective epidemiological research studies in Iran (the PERSIAN Cohort Study): rationale, objectives, and design. Am J Epidemiol. 2017; 187(4):647-55.

17. Rajati F, et al. Prevalence, awareness, treatment, and control of hypertension and their determinants: results from the first cohort of non-communicable diseases in a Kurdish settlement. Sci Rep. 2019;9(1):1-10.

18. Safari-Faramani $R$, et al. Prevalence, awareness, treatment, control, and the associated factors of diabetes in an Iranian Kurdish population. J Diabet Res. 2019;2019:1.

19. Najafi F, et al. The reliability of self-reporting chronic diseases: how reliable is the result of population-based cohort studies. J Prevent Med Hygiene. 2019;60(4):E349.

20. Ashrafi S, et al. The validity of self-reported drug use with urine test: results from the pilot phase of Azar cohort study. Health Promot Perspect. 2018;8(3):225.

21. Association AD. American Dental Association Statement on Regular Brushing and Flossing to Help Prevent Oral Infections; 2013.

22. Eslamipour F, Borzabadi-Farahani A, Asgari I. The relationship between aging and oral health inequalities assessed by the DMFT index. Eur J Paediatr Dent. 2010;11(4):193. 
23. Gh FM. Evaluation of the reliability and validity of Azad-Fesharaki's physical activity questionnaire (AFPAQ). Arak Med Univ J. 2011;14(56):36-44.

24. Werner S, Malaspina D, Rabinowitz J. Socioeconomic status at birth is associated with risk of schizophrenia: population-based multilevel study. Schizophr Bull. 2007;33(6):1373-8.

25. Mohammadifard N, et al. Validation of a simplified food frequency questionnaire for the assessment of dietary habits in Iranian adults: Isfahan Healthy Heart Program, Iran. ARYA Atherosclerosis. 2015;11(2):139.

26. Mirzaei M, et al. Psychometric evaluation of a self-reported physical activity questionnarie used in the pilot phase of the AZAR Cohort Study. Health Promot Perspect. 2016;6(3):152.

27. Ghassemi H, Harrison G, Mohammad K. An accelerated nutrition transition in Iran. Public Health Nutr. 2002;5(1a):149-55.

28. Vander Weele TJJAROPH. Mediation analysis: a practitioner's guide, vol. 37. Oxford: Oxford University Press; 2016.

29. Deonandan RS. m VITRO FERTILIZATION; 2000

30. Robins JM. Marginal structural models versus structural nested models as tools for causal inference. In: Statistical models in epidemiology, the environment, and clinical trials. Berlin: Springer; 2000. p. 95-133.

31. VanderWeele TJ. Mediation analysis: a practitioner's guide. Annu Rev Public Health. 2016;37:17-32.

32. Chauvet-Gelinier J-C, Bonin B. Stress, anxiety and depression in heart disease patients: a major challenge for cardiac rehabilitation. Ann Phys Rehab Med. 2017;60(1):6-12.
33. Eichstaedt JC, et al. Psychological language on Twitter predicts countylevel heart disease mortality. Psychol Sci. 2015;26(2):159-69.

34. Forouzanfar MM, et al. Short-term outcome of elderly patients discharged from an emergency department; 2013.

35. Mohammadi $M$, et al. An epidemiological study of psychiatric disorders in Iran, 2001; 2003.

36. Sadeghi KA, Asareh M. Epidemiological study of psychiatric disorder in Kermanshah Urban residents; 2000.

37. Beukers NG, et al. Periodontitis is an independent risk indicator for atherosclerotic cardiovascular diseases among 60174 participants in a large dental school in the Netherlands. J Epidemiol Community Health. 2017;71(1):37-42.

38. Ivanovs R. Association of depression and anxiety with cardiovascular co-morbidity and 10-year risk of cardiovascular mortality (SCORE) in a primary care population of Latvia. Summary of the Doctoral Thesis; 2020.

39. Whooley MA, Wong JM. Depression and cardiovascular disorders. Annu Rev Clin Psychol. 2013;9:327-54

\section{Publisher's Note}

Springer Nature remains neutral with regard to jurisdictional claims in published maps and institutional affiliations.
Ready to submit your research? Choose BMC and benefit from:

- fast, convenient online submission

- thorough peer review by experienced researchers in your field

- rapid publication on acceptance

- support for research data, including large and complex data types

- gold Open Access which fosters wider collaboration and increased citations

- maximum visibility for your research: over $100 \mathrm{M}$ website views per year

At BMC, research is always in progress.

Learn more biomedcentral.com/submissions 